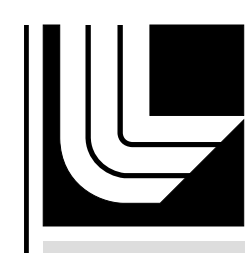

LA W RENCE LIVERM ORE NATIONAL LABORATORY

\title{
CALIBRATION OF X-RAY IMAGING DEVICES FOR ACCURATE INTENSITY MEASUREMENT
}

M. J. Haugh, M. R. Charest, P. W. Ross, J. J. Lee, M. B. Schneider, N. E. Palmer, A. T. Teruya

February 21, 2012

Denver X-Ray Conference Denver, CO, United States August 1, 2011 through August 5, 2011 
This document was prepared as an account of work sponsored by an agency of the United States government. Neither the United States government nor Lawrence Livermore National Security, LLC, nor any of their employees makes any warranty, expressed or implied, or assumes any legal liability or responsibility for the accuracy, completeness, or usefulness of any information, apparatus, product, or process disclosed, or represents that its use would not infringe privately owned rights. Reference herein to any specific commercial product, process, or service by trade name, trademark, manufacturer, or otherwise does not necessarily constitute or imply its endorsement, recommendation, or favoring by the United States government or Lawrence Livermore National Security, LLC. The views and opinions of authors expressed herein do not necessarily state or reflect those of the United States government or Lawrence Livermore National Security, LLC, and shall not be used for advertising or product endorsement purposes. 


\title{
CALIBRATION OF X-RAY IMAGING DEVICES FOR ACCURATE INTENSITY MEASUREMENT
}

\author{
Michael J. Haugh*, Michael Charest*, Patrick Ross*, Joshua Lee*, \\ Marilyn Schneider", Nathan Palmer ${ }^{\star}$, and Alan Teruya \\ *National Security Technologies, LLC, 161 S. Vasco Rd. Suite A, Livermore CA 94550 \\ •Lawrence Livermore National Laboratory, 7000 East Ave., Livermore, CA 94550
}

\begin{abstract}
National Security Technologies (NSTec) has developed calibration procedures for X-ray imaging systems. The X-ray sources that are used for calibration are both diode type and diode/fluorescer combinations. Calibrating the X-ray detectors is key to accurate calibration of the X-ray sources. Both energy dispersive detectors and photodiodes measuring total flux were used. We have developed calibration techniques for the detectors using radioactive sources that are traceable to the National Institute of Standards and Technology (NIST). The German synchrotron at Physikalische Technische Bundestalt (PTB) is used to calibrate silicon photodiodes over the energy range from $50 \mathrm{eV}$ to $60 \mathrm{keV}$.
\end{abstract}

The measurements on X-ray cameras made using the NSTec X-ray sources have included quantum efficiency averaged over all pixels, camera counts per photon per pixel, and response variation across the sensor. The instrumentation required to accomplish the calibrations is described. X-ray energies ranged from $720 \mathrm{eV}$ to $22.7 \mathrm{keV}$. The X-ray sources produce narrow energy bands, allowing us to determine the properties as a function of X-ray energy. The calibrations were done for several types of imaging devices. There were back illuminated and front illuminated CCD (charge coupled device) sensors, and a CID (charge injection device) type camera. The CCD and CID camera types differ significantly in some of their properties that affect the accuracy of X-ray intensity measurements. All cameras discussed here are silicon based. The measurements of quantum efficiency variation with X-ray energy are compared to models for the sensor structure. Cameras that are not back-thinned are compared to those that are.

\section{INTRODUCTION}

Historically, X-ray imaging has been used to determine geometric and qualitative intensity information, such as tooth damage or star luminosity. For many endeavors, including astronomy, non-destructive testing, medicine, and plasma physics, the ability to measure accurately the X-ray intensity in a specific energy interval has become more important. But the metrology for these measurements, namely the traceability to an internationally accepted standard, is not well developed. Over the past five years, National Security Technologies, LLC (NSTec) has built up several laboratories and developed X-ray sources to cover measurements in the energy range from $50 \mathrm{eV}$ to $115 \mathrm{keV}$. These sources are used to calibrate measurement systems (usually referred to as diagnostics) and diagnostic components that are used for plasma studies at the Lawrence Livermore National Laboratory (LLNL) and the National Ignition Facility (NIF) at LLNL. This paper describes two X-ray sources that are used to calibrate 
imaging devices. To use these X-ray sources for calibrating imaging devices, it is necessary to know the X-ray beam spectrum, the photon flux, and the flux distribution throughout the beam area. This is accomplished using energy dispersive solid state detectors and photodiodes. The calibration of these detectors to international standards is described. These sources have been used to calibrate several types of imaging devices. This includes image plates and X-ray cameras. The image plate work has been published (Maddox, 2001). This paper describes the calibration of several types of X-ray cameras: back illuminated and back thinned CCD cameras, front illuminated CCD cameras, and Charge Injection Device (CID) type X-ray cameras.

\section{X-RAY SOURCES}

\section{The HEX Source}

The High Energy X-ray source (HEX) generates spectral lines in a beam using a commercial $160 \mathrm{kV}$ tungsten $\mathrm{X}$-ray tube with fluorescing targets that enclosed in a lead box. An exit collimator in the lead box shapes the X-ray beam. The arrangement of the components is shown in Figure 1.

The end of the commercial X-ray tube is shown in yellow. The pink trapezoid that starts at the tube represents the primary Xray beam. The fluorescers are mounted on the motorized wheel in the rectangles shown

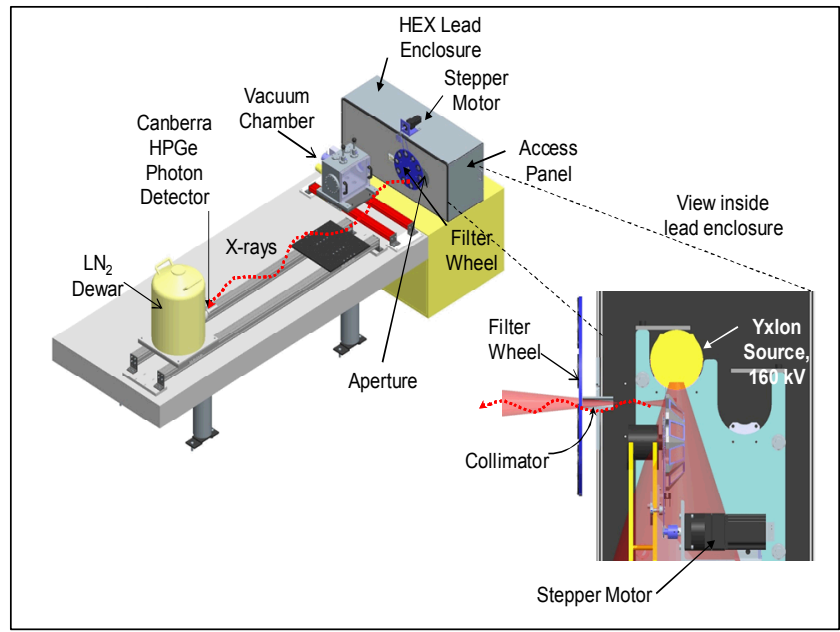

Figure 1. The HEX system layout and the fluorescer source arrangement. on the wheel. The fluorescer emits in all directions, but the X-ray beam is defined by the collimator inserted into the wall of the lead box, and the beam path is illustrated by the pink triangles. There is a filter wheel mounted downstream from the collimator, and it is also motorized. The fluorescer and the filter can be set from the computer in an adjacent control room. The fluorescer is usually a thin sheet made of elemental metal, but metal compounds are sometimes used. The maximum flux obtained is on the order of $4 \times 10^{6}$ photons per $\mathrm{cm}^{2}$ per second, at one meter from the fluorescer, depending on the fluorescer material. The available spectral lines from this source range from $6 \mathrm{keV}$ to $115 \mathrm{keV}$.

The HEX source sits at the end of an optical table as shown in Figure 1. The sample, such as a CCD camera, and the detectors are mounted on the optical table.

A typical spectrum from the HEX is shown in

$\mathrm{Pb}$ Spectrum, Pt Filter

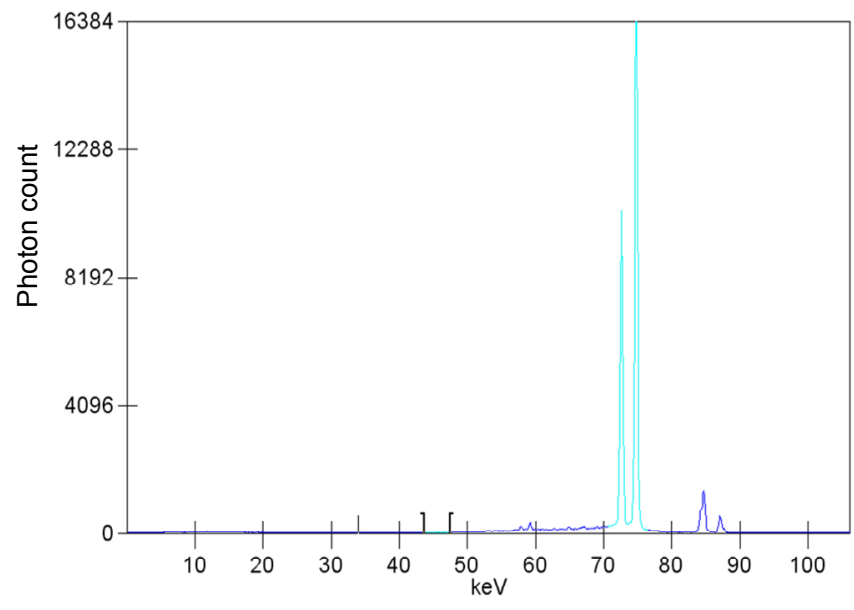

Figure 2. A typical spectrum from the HEX source observed using a solid state Ge energy dispersive detector.

Figure 2. For this example, the fluorescing material is a thin lead $(\mathrm{Pb})$ sheet, with a thickness of 
approximately $250 \mu \mathrm{m}$, and the filter is a thin platinum $(P t)$ sheet. The high energy X-ray lines are transmitted by the filter, but the low energy lines are stopped by the filter. This method provides a reasonably narrow spectral energy that can be used to calibrate detectors at a range of well defined energies.

\section{The Medium Resolution X-ray Source (MRXS); a Diode Source}

The MXRS is a diode type X-ray source that is used for testing and calibrating various devices. It is a multiple anode source so that the X-ray spectral lines can be selected, as needed for the task, in the spectral energy range from $400 \mathrm{eV}$ to $9 \mathrm{keV}$. Figure 3 shows a schematic diagram of the NSTec MRSX system, looking down on it from above. The MRXS comprises three independent vacuum compartments: the source chamber, which is the circle, and two test chambers which are the rectangular boxes in the figure.

The source chamber contains the array of anodes, the filament, and filters. The array can be rotated putting any one of the 6 anode materials in front of the filament. The X-rays emitted from the operating anode are formed into 2 beams that travel into the test chambers. The anode is not water cooled, and the power is limited to $10 \mathrm{~W}$ to avoid melting the anodes. A wide variety of anode materials are used. The filament is shaped to a point near the anode. This produces a small spot, approximately $1 \mathrm{~mm}$ diameter, where the electrons impact the anode. This small Xray emission spot acts as a point source providing a consistent X-ray intensity in the sample region. This provides a flat field image, allowing us to measure the sensitivity variation across the sensor array of a camera.

The two test chambers are connected to the main chamber by stainless steel vacuum components that include an isolation gate valve and a mechanical shutter. The diagnostic that is shown attached to the top arm in the figure is at vacuum. Components such as filters can be mounted inside the chamber.

Each test chamber has its own vacuum pump and controls and can be isolated from the source chamber by a gate valve, then brought to atmosphere. Test

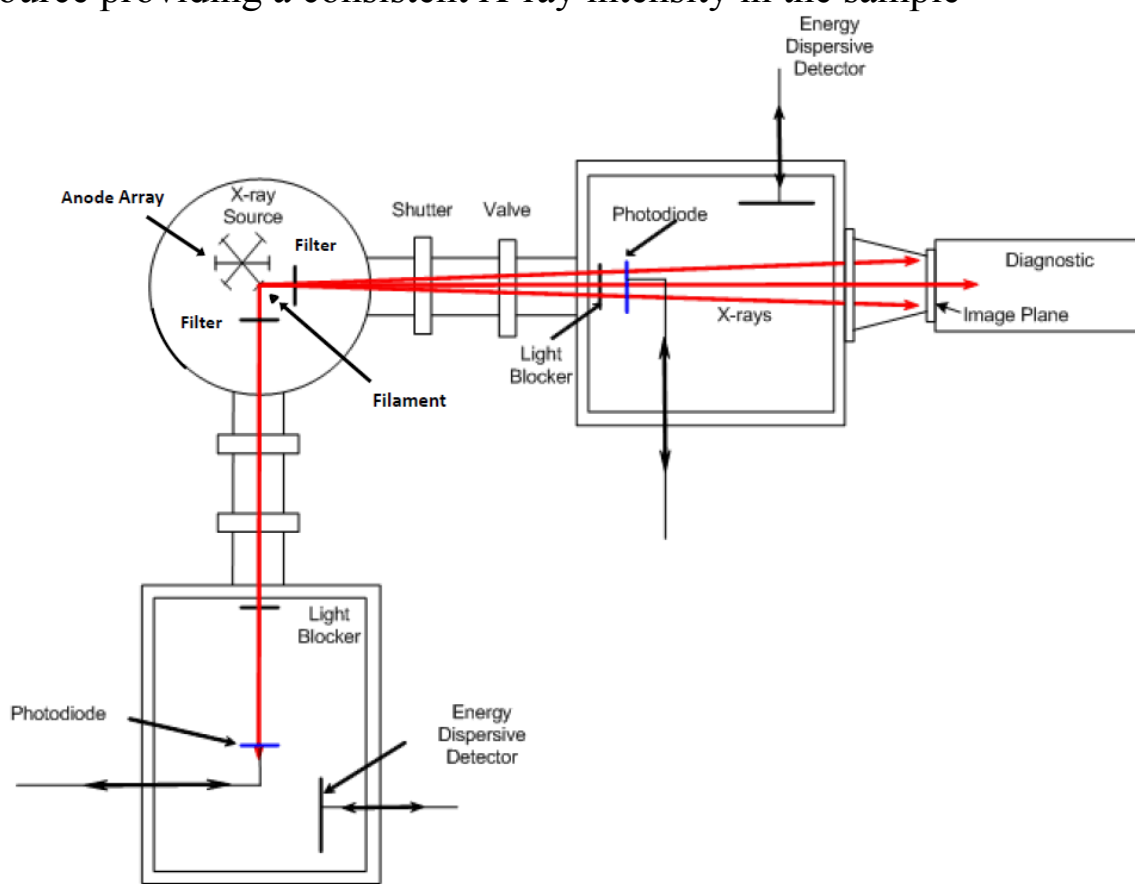

Figure 3. Schematic diagram of the MRXS comprising three separate vacuum chambers. The multiple diode source is in the center chamber. chambers have a photodiode and an energy dispersive detector for measuring X-ray flux and the $\mathrm{X}$-ray spectrum, mounted on push rods so that they can be moved into or out of the beam. 
The X-ray beam paths that are used for testing are shown as red lines in Figure 3. The filters, shown in the source chamber, are used to isolate a narrow wavelength band of $\mathrm{X}$-rays. These filters are mounted in a vertical stalk that holds up to three filters. A light blocker prevents visible light emitted by the filament from entering the test chamber which would overwhelm the photodiode and CCD.

Figure 4 shows the Ti spectrum that is produced when using a Ti anode and having a $100 \mu \mathrm{m}$ thick Ti filter in the beam path which acts as a band pass filter. We can see that the transmission is limited to the spectral energy range between $4300 \mathrm{eV}$ and $4966 \mathrm{eV}$, the latter energy being the $\mathrm{K}$ edge of $\mathrm{Ti}$. The spectral content now includes the $\mathrm{Ti} \mathrm{K}_{\alpha}$ lines (the large peak), the $\mathrm{Ti} \mathrm{K}_{\beta}$ lines, and the bremsstrahlung within the energy range given.

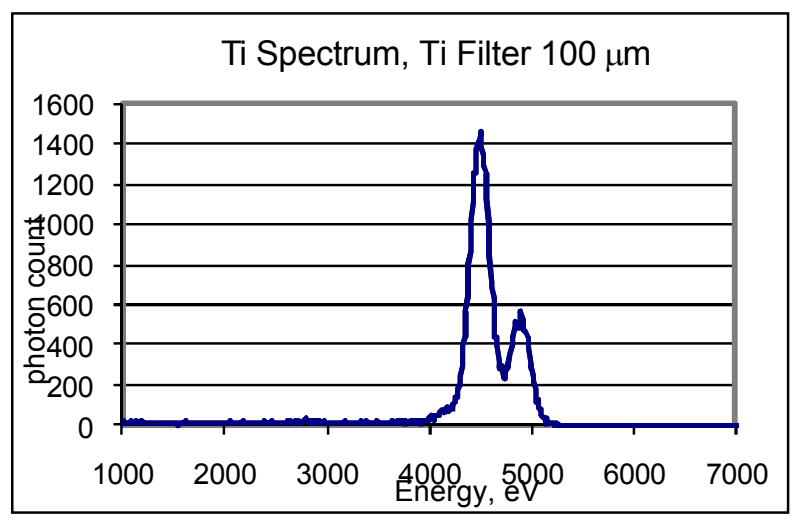

Figure 4. The $\mathrm{X}$-ray spectrum obtained using a $\mathrm{Ti}$ anode and a Ti filter to produce an X-ray beam comprising mainly the $\mathrm{Ti} \mathrm{K}_{\alpha}$ and $\mathrm{K}_{\beta}$ spectral lines. It is observed using a Si solid state energy dispersive detector.

\section{CALIBRATING THE DETECTORS}

The X-ray sources are used to calibrate X-ray imaging devices. The X-ray source spectrum, flux, and flux distribution in the beam are measured with calibrated detectors. The following section gives a brief description of solid state detectors and describes the calibration technique.

\section{Introduction to semiconductor detectors}

In the field of X-ray measurements, the term solid state detector usually refers to semiconductor type detectors. There are other X-ray detectors that are solid materials, such as scintillator photomultiplier combinations, but for this paper the term solid state detectors will only refer to semiconductor detectors. There are 3 types of semiconductor materials used for the work described in this paper: silicon ( $\mathrm{Si}$ ), germanium $(\mathrm{Ge})$, and cadmium telluride $(\mathrm{CdTe})$. A semiconductor is defined as a material that has a small band gap (which can be manipulated) between the valence electrons and the conduction band, on the order of $1 \mathrm{eV}$ to several $\mathrm{eV}$.

Consider an X-ray photon incident on the semiconductor. It has an energy that is many times that of the band gap. It interacts with the semiconductor material, primarily through the photoelectric effect, to produce energetic free electrons. These then produce electron-hole pairs. The number of electron-hole pairs produced is proportional to the energy of the X-ray photon as shown in Table 1. Not all photons impinging the sensor are detected. The number of photons detected divided by the number of photons incident on the detector is called the quantum efficiency. Details of semiconductor behavior and their application to X-ray intensity measurement can be found in Knoll (2001) and Haugh (2011). 
Table 1. Properties of semiconductors discussed in this section

\begin{tabular}{|c|c|c|}
\hline $\begin{array}{c}\text { Semiconductor } \\
\text { material }\end{array}$ & $\begin{array}{c}\text { \&, Energy required } \\
\text { for one hole/pair } \\
\text { production, eV }\end{array}$ & $\begin{array}{c}\text { Semiconductor } \\
\text { band gap, eV }\end{array}$ \\
\hline $\mathrm{Si}$ & 3.63 & 1.116 \\
\hline $\mathrm{Ge}$ & 2.96 & 0.665 \\
\hline $\mathrm{CdTe}$ & $4.43^{*}$ & 1.44 \\
\hline
\end{tabular}

*(Quaranta, 1969)

There are several ways that the signal can be lost and the solid state detector output fails to give the full measure of the X-ray intensity. For this reason the detectors must be calibrated.

Calibrated photodiodes are used to measure the X-ray flux of X-ray sources. The silicon photodiodes used for this work were designed to measure X-ray intensity with nearly $100 \%$ quantum efficiency for up to X-ray energies of about $5 \mathrm{keV}$ (International Radiation Detectors Reference). Several of these photodiodes have been calibrated from $1000 \mathrm{eV}$ to $60 \mathrm{keV}$ at the Physikalisch-Technische Bundensanstalt (PTB Reference; Gottwald, 2006). Within the measurement uncertainty, which is near $1 \%$, the silicon photodiode is $100 \%$ effective up to the $\mathrm{Si}$ $\mathrm{K}$ edge, where it dropped several percent. The efficiency rises back to near $100 \%$ by $3 \mathrm{keV}$. The Si photodiode is $55 \mu \mathrm{m}$ thick and begins to transmit X-rays near $5 \mathrm{keV} \mathrm{X}$-ray energy. At higher energies the diode efficiency follows the Si transmission curve.

Energy dispersive detectors are designed to measure the energy spectrum of the X-ray photons. A bias voltage is applied to the semiconductor and the electric fields generated require cooling of the detector. The voltage pulses produced by an individual X-rays are amplified and then counted according to pulse height. Pulses that have heights within a certain range are effectively assigned to channels according to the average pulse height by a processor referred to as a multichannel analyzer. This produces an energy spectrum of the detected X-ray photons. The resolving power of the energy dispersive detector is generally limited to several hundred $\mathrm{eV}$.

The detector sensitivity falls off at lower energies due to absorption at the front surface by a "dead" layer of the sensor and/or a window separating the vacuum chamber containing the detector from the environment. It also falls off at higher energy when the photons begin to be transmitted by the sensor.

\section{Detector Calibration Results Using Radioactive Sources}

Radioactive sources provide a variety of spectral lines at well defined energies. The photon output is directly proportional to the activity of the radioactive source, and the activity measurement is traceable to NIST. The uncertainty for the activity is provided by the vendor. The spectral lines that were used for these measurements are given in Table 2. 
Table 2. Spectral Lines Used for the Calibration of the Ge and CdTe Detectors

\begin{tabular}{|c|c|c|}
\hline Spectral Energy, keV & Spectral Type & Radionuclide \\
\hline 5.97 & $\mathrm{Mn}, \mathrm{K}$ line & $\mathrm{Fe} 55$ \\
\hline 22.16 & $\mathrm{Ag}, \mathrm{K}$ line & $\mathrm{Cd} 109$ \\
\hline 26.34 & $\gamma$ & $\mathrm{Am} 241$ \\
\hline 41.3 & $\mathrm{Eu}, \mathrm{K}$ line & $\mathrm{Gd} 153$ \\
\hline 59.54 & $\gamma$ & $\mathrm{Am} 241$ \\
\hline 70.0 & $\gamma$ & $\mathrm{Gd} 153$ \\
\hline 88.00 & $\gamma$ & $\mathrm{Cd} 109$ \\
\hline 97.43 & $\gamma$ & $\mathrm{Gd} 153$ \\
\hline 103.18 & $\gamma$ & $\mathrm{Gd} 153$ \\
\hline
\end{tabular}

Quantum efficiency measurements for the Canberra HP Ge detector (Canberra Ultra-LEGe, GUL0055) are shown in Figure 5. This graph shows the reproducibility of the calibration measurements as done independently by two operators. The $95 \%$ confidence interval for the detector quantum efficiency is near $4 \%$.

The situation regarding the large gaps can be improved by using more radionuclides but there are availability and economic restrictions that will limit this approach. We plan to fill in these energy gaps by calibrating the Si detector (IRD, AXUV-100GX-G) up to $60 \mathrm{keV}$ using a synchrotron as described in the next sub-section. This calibration can then be transferred to the other detectors using the HEX source.

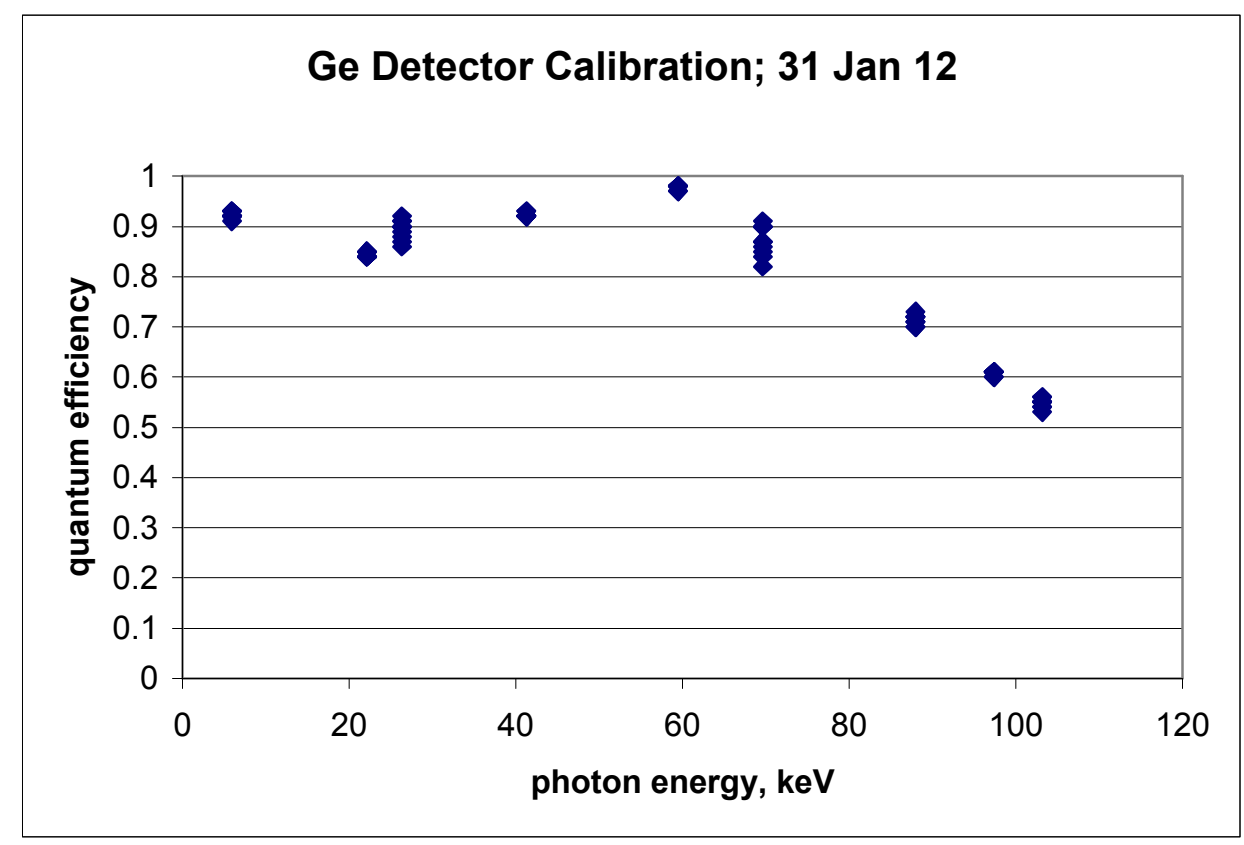

Figure 5. Quantum efficiency measurements for the Ge detector 


\section{MEASURING THE QUANTUM EFFICIENCY AND ITS SPATIAL VARIATION FOR X-RAY CAMERAS}

\section{Analysis and Imaging Procedures}

The X-ray photon interacts with the Si sensor to produce electron-hole pairs that the camera electronics process to produce the digital signal count $\mathrm{S}$. The number of electron-hole pairs produced by an X-ray photon that interacts with the Si sensor is a function of the photon energy and is slightly dependent upon the temperature (Janesick, 2000). The sensor is cooled to 253K when operating.

The camera sensitivity, $\mathrm{S}(\mathrm{E})$, is defined as the number of camera counts produced by the absorption of a photon of energy E. The X-ray beam flux is measured using one of the detectors described previously and the camera signal is measured in the same beam. The camera sensitivity is then given by:

$$
\mathbf{S}(\mathbf{E})=\mathbf{C} /\left(\mathbf{P} \bullet \mathbf{A}_{\text {pix }} \bullet t\right) \quad \text { counts } / \text { photon }
$$

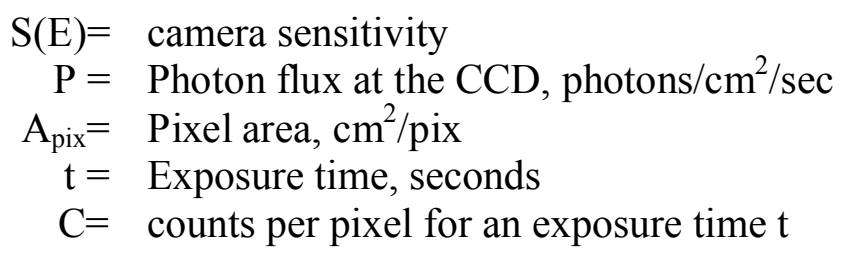

A useful model relating the camera signal to fundamental quantities is found in Janesick (2000).

$$
\mathbf{S}(\mathbf{E})=\mathbf{P} \bullet \mathbf{A}_{\mathbf{p i x}} \bullet \mathbf{t} \bullet \eta \cdot \mathbf{Q E} \cdot \mathbf{K}^{-1} \text { counts/photon }
$$

$\mathrm{QE}=$ Quantum efficiency, fraction of photons that interact with the pixel

$\eta=$ Quantum yield, number of electron-hole pairs produced by the photon

$\mathrm{K}=$ Camera gain, electron-hole pair per count

For the Si CCD, the quantum yield is given by (Knoll, 2001):

$$
\eta=\mathbf{E} / \mathbf{3 . 6 6} \text { hole pairs/photon }
$$

where $\mathrm{E}$ is the energy of the photon in $\mathrm{eV}$.

The quantity being determined by the calibration is $\mathrm{S}(\mathrm{E})$, as described in the previous paragraph, but the model given by Equation 2 can be used to calculate the QE. The QE provides a valuable check of the calibration procedure, the calibration implementation, and is useful for troubleshooting camera problems. For the Si based cameras, the QE is related to the properties of $\mathrm{Si}$ as we shall see in the following results.

For measurements made using the MRXS, the photon flux $\mathrm{P}$ was measured using the $\mathrm{Si}$ photodiode manufactured by IRD, described in the detector section. The photon intensity was measured with the photodiode in the top arm as seen in Figure 3. The Ge detector was used for measurements made on HEX. An exposure time, on the order of several minutes, was chosen to be as short as possible to give a reasonable signal. Photodiode readings were taken before and after acquiring each CCD image. The camera was mounted on the diagnostic arm as shown in Figure 3. There was an extension between the camera and the MRXS chamber of 
sufficient length that the X-ray beam uniformly illuminated the camera sensor. During imaging, the X-ray beam intensity was monitored continuously for beam fluctuations using the photodiode in the lower arm. If there were beam intensity fluctuations observed during imaging, that image was discarded.

The cameras evaluated had large numbers of bad rows and hot pixels. The bad rows were associated with the readout and identified using closed shutter images with a $3 \mathrm{~ms}$ exposure time. The hot pixels were identified by taking a short exposure image using the Ti anode and no filter. A map was made that identified the bad rows and bad pixels.

The camera images for the efficiency analysis had the background (closed shutter images) subtracted and the bad pixels replaced by the average of adjacent pixels. The mean pixel count was determined by randomly selecting 1000 regions $20 \times 20$ pixels in size, calculating the mean counts/pixel for each region and calculating the average of the means for each region. This is the signal $\mathrm{S}(\mathrm{E})$ for that image.

Flat field images are images where the CCD is uniformly illuminated in order to measure the uniformity of the camera response over its area. They were taken using the same anode voltage that was used for the camera efficiency measurements and with maximum anode current. The exposure time was chosen to produce a signal that was $50 \%$ to $60 \%$ of saturation. Multiple flat field images and background images (shutter closed) were taken at each photon energy in order to reduce photon statistical noise. The flat field images and the background images were averaged and then the average background subtracted from the average flat field image.

\section{MEASUREMENT RESULTS}

\section{A Back Illuminated, Back Thinned Large Format CCD Camera}

The SXI (Static X-ray Imager) cameras are large format $(2 \mathrm{kx} 2 \mathrm{k}, 24 \mu \mathrm{m}$ square pixels), back thinned and back illuminated. They are used on the NIF target chamber. The quantum efficiency for one of the SXI cameras is given in Figure 6. The Quantum Efficiency $(\mathrm{QE})$ calculated from the camera signal using Eq. 2 and 3 with camera gain $\mathrm{K}=7.62$ electron-hole pair per count is plotted as a function of photon energy. The measurements below $9 \mathrm{keV}$ were done on the MRXS; the higher energy

measurements were done on the HEX. The dip near $1800 \mathrm{eV}$ and the fall-off after $2000 \mathrm{eV}$ are caused by properties of $\mathrm{Si}$. The Si CCD for the SXI camera is $15 \mu \mathrm{m}$ thick. Si that is $15 \mu \mathrm{m}$ thick transmits up to $35 \%$ as it approaches the $\mathrm{K}$ edge at $1839 \mathrm{eV}$. It begins transmitting again above $2500 \mathrm{eV}$ and is transmitting $80 \%$ at

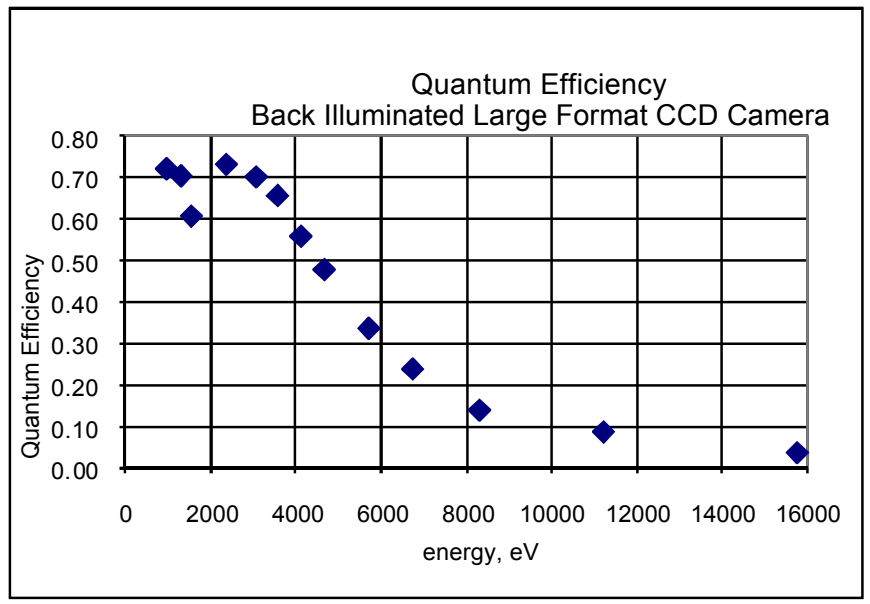

Figure 6. The SXI quantum efficiency was measured using the average camera count per pixel for each photon of a given energy. The measurements made at X-ray energies below $8800 \mathrm{eV}$ were done on the Manson. The higher energy measurements were done on the HEX. 
$8 \mathrm{keV}$. These QE results are similar to that obtained by Poletto (1999). There are two possible causes why the QE does not approach 1 when the photons are completely absorbed: (1) There may be absorption at the surface coating of the $\mathrm{Si}$; (2) the Quantum Yield may be less than the photon energy divided by $3.66 \mathrm{eV}$ per electron-hole pair. Analysis of a large number of single photon events could show the relative contribution of each effect.

The flat field X-ray source is the $1 \mathrm{~mm}$ diameter spot formed by the filament electron beam from the filament hitting the MRXS anode. The anode is $1405 \mathrm{~mm}$ from the CCD. This arrangement would produce a flat field within $1 \%$ if there were nothing between the anode and the CCD. There is a light blocker that has an aluminum coating on a polyimide film (Al $1054 \AA \pm 50 \AA$; polyimide $1081 \AA \pm 100 \AA$ ). This item does not affect the flat field within the $1 \%$ cited above. The filter can cause a variation in the beam intensity across the CCD if there is sufficient variation in thickness, foreign material, or misalignment with the anode.

Figure 7 shows the flat field image for one of the SXI cameras at the $\mathrm{Cu} 8470 \mathrm{eV}$ energy band

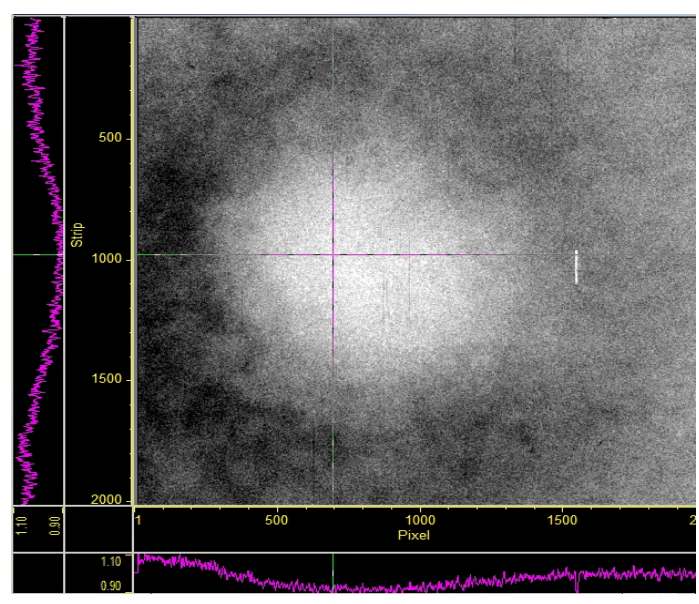

Figure 7. Flat field image for one of the SXI cameras at the $\mathrm{Cu} 8470 \mathrm{eV}$ energy

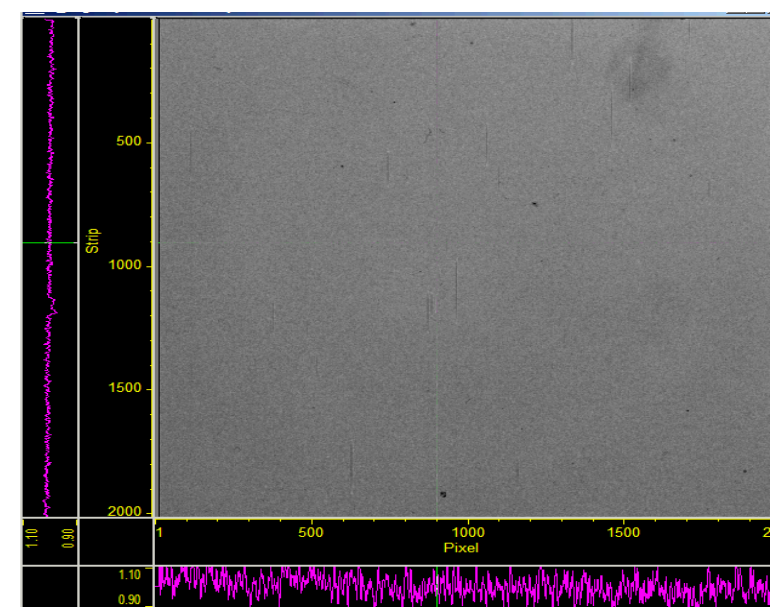

Figure 8. This is a flat field image using the $\mathrm{Mg}$ $1273 \mathrm{eV}$ spectral band. It is completely uniform within $1 \%$; the high energy non-uniformity has completely disappeared.

when the MXRS $\mathrm{Cu}$ anode is selected. The image is set at high contrast so that the pixel signal variation shows clearly. A gross pattern is observed with the sensitivity at a maximum near the left center and decreasing slowly going away from the maximum. The maximum sensitivity variation is $10 \%$. The image in Figure 8 is obtained using the Mg anode selection of the MXRS producing $1273 \mathrm{eV} \mathrm{X-}$ rays. The sensitivity variation observed at the higher energies is completely gone at this lower energy.

A different phenomenon was seen at low energies. Small irregular patches having diminished sensitivity were observed that are readily seen in Figure 9. This image

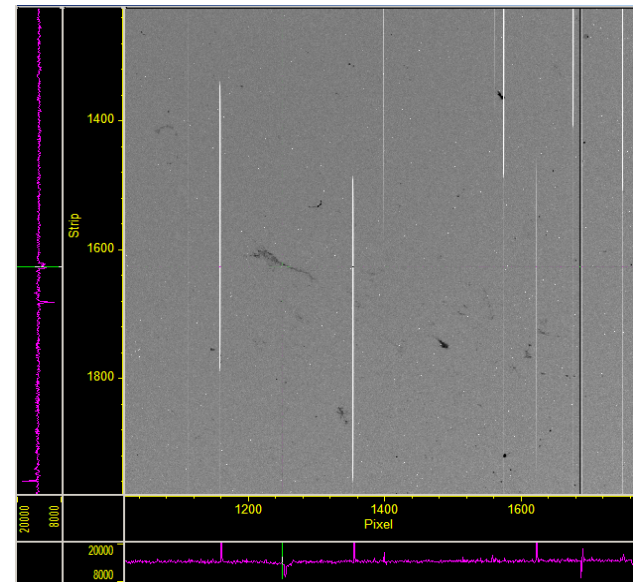

Figure 9. Damaged region of SXI $\mathrm{CCD}, 1273 \mathrm{eV}$ 
shows a portion of the CCD. The effect on sensitivity in these regions also shows an energy dependence. The irregular patches become less pronounced as the spectral energy increases. At $4500 \mathrm{eV}$, these patches have completely disappeared.

There are several possible causes for these dark regions. Debris on the CCD surface could absorb X-rays and would be energy dependent, absorbing X-rays less as the energy increased. Damage to the CCD Would likely cause an energy dependence that would increase the variance of the defective region from the surrounding pixels as the energy increased. Damage to the surface coating could produce this effect if the coating were thicker in that defective region. When we examined the CCD surface with a magnifying glass it did appear that the coating was deformed. It looked like a manufacturing defect.

\section{Calibrating a Front Illuminated CCD Camera}

The SXI camera described above plays a critical role in the NIF operation, but this specific chip is no longer manufactured. There is another chip on the market with this large array, $2 \mathrm{kx} 2 \mathrm{k}, 24$ $\mu \mathrm{m}$ square, and we received a request to test the chip in a standard camera. The major concern regarding this chip was that it is front illuminated.

The QE measurements were made at $\mathrm{X}$-ray energies ranging from $710 \mathrm{eV}$ to $16 \mathrm{keV}$. These measurements are shown in the graph of Figure 10. Compare this to the results shown in Figure 6 for the QE of the back illuminated camera. The maximum $\mathrm{QE}$ for the front illuminated camera

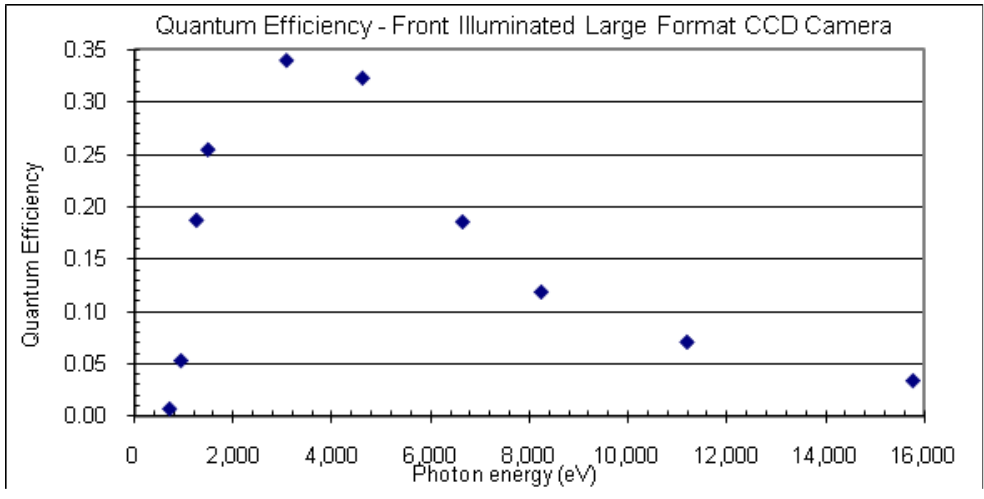

Figure10. Quantum Efficiency, Front Illuminated Large Format CCD Camera is $\mathrm{QE}=0.34$ near $2300 \mathrm{eV}$. This is a factor of 2 lower than the $\mathrm{QE}$ measured for the back illuminated camera. The predominant difference begins to show below $1000 \mathrm{eV}$. At the $\mathrm{Cu} \mathrm{L}$ lines, near $930 \mathrm{eV}$, the QE for the front illuminated camera is down by a factor of 10 from the front illuminated camera. At the Fe L lines near $710 \mathrm{eV}$, the QE is down by a factor of 100 . At energies above $10 \mathrm{keV}$, the two types of camera have similar quantum efficiencies.

\section{Characterizing and Calibrating an Uncooled X-ray CID (Charge Injection Device) Camera Using the HEX Source}

This section describes the characterization of a CID camera. The camera is the detector in a spectrometer system that is used on the LLNL NIF target chamber. The initial interest was to measure the emission from highly ionized Ge so the camera was characterized in the $10 \mathrm{keV}$ region using the HEX source (Carbone, 1998; Marshall, 2001). The fluorescers chosen were $\mathrm{Cu}$, $\mathrm{Ge}$, and $\mathrm{Rb}$ giving weighted average for the $\mathrm{K}-\mathrm{L}$ and $\mathrm{K}-\mathrm{M}$ transitions of $8.13 \mathrm{keV}, 10.01 \mathrm{keV}$, and $13.58 \mathrm{keV}$ respectively.

The major use for this type of CID sensor is for dental X-rays. It is relatively cheap and therefore expendable, a desirable property for the NIF application. The camera operates at room temperature normally, which gave a challenging problem to the characterization on HEX. Since 
the CID operates at room temperature, the dark current can saturate the camera for exposure times greater than 5 seconds. This not a problem on NIF since the exposure time can be less than 1 second with sufficient X-rays to provide a bright spectral image.

As indicated in the earlier description of CCD camera calibrations on the MRXS, minutes of exposure time are needed to get a satisfactory signal. Preliminary experiments with the CID camera showed that we would be limited to three-second exposure times. It was determined that multiple exposures, on the order of 50 exposures, would be needed to obtain satisfactory images for calibrating the CID. The multiple exposures allow us to average the readout noise, which is quite high in this device. A shutter control system was implemented for automatically taking the multiple images. We quickly found that drift in the dark current required us to take background images immediately after the X-ray exposure. The system was designed so that an image was taken with the shutter open to the X-rays, then the next image was taken with the shutter closed. In this way a pair of images were produced, one image exposed to X-rays and the other as a background, close enough in time that there was no observable dark current drift. A black Kapton sheet, $50 \mu \mathrm{m}$ thick, was used to shield the camera from visible light. The same type shield is used for the camera on the NIF target chamber.

The X-ray beam was characterized geometrically using image plates to optimize collimator and distance choices. The intensity distribution was measured using the CdTe energy dispersive detector at multiple locations across the beam. Multiple images were taken with the CID, and then the detector was placed at the same location as the center of the CID had been located to verify that there was no drift in the X-ray source intensity. The multiple images were analyzed by subtracting each background from the previously taken $\mathrm{X}$-ray image and summing the 50 resulting images. The final image then was effectively a 150 second exposure with the background removed. Figure 11 shows a typical image resulting from this process. The measurements concentrated on the X-ray beam center for this initial effort. The CID camera sensitivity, counts per photon, could then be calculated using the CdTe intensity measurements. The individual horizontal rows seen in the image result from the camera read out noise.

The results are shown in Figure 12. The camera response was measured for two CID cameras at three spectral energies over the range of interest. The responses of the two cameras are the same within the experimental uncertainty. The expected response was modeled using the vendor's specification for camera gain, Si thickness, and a typical surface coating. This is shown by the blue

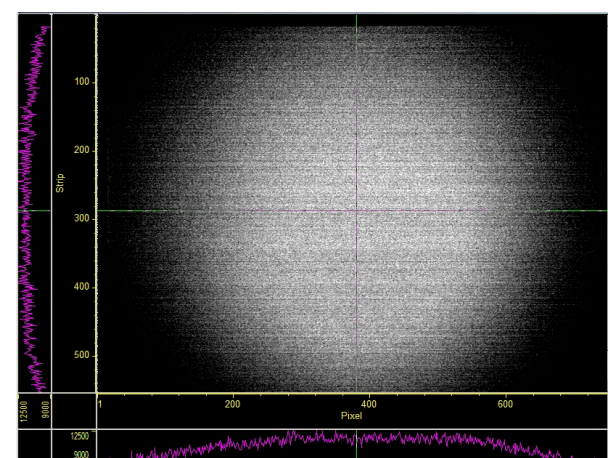

Figure 11. An image of the HEX $\mathrm{X}$-ray beam from a Charge Injection Device (CID) camera that is the sum of 50 individual images.

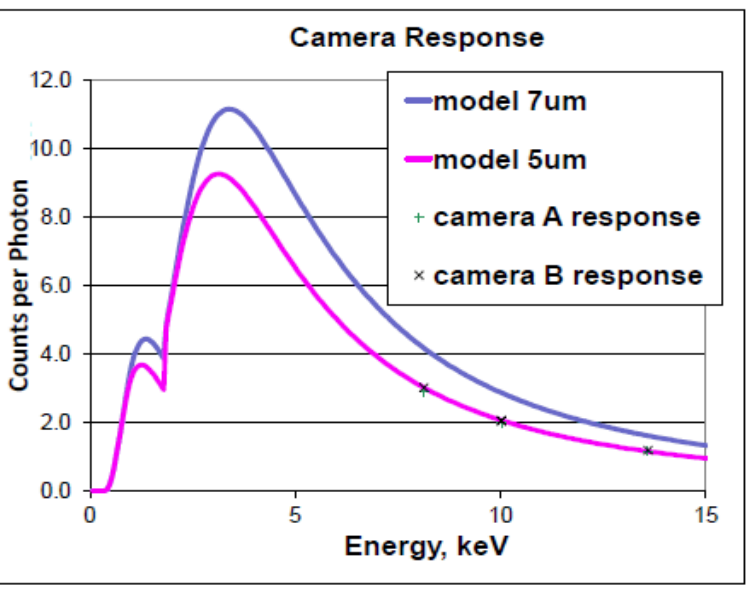

Figure 12. Camera sensitivity for two CID cameras. The curves are models for the camera response. 
line in the figure. This did not fit the measurement data so a second model curve is shown using a thinner Si effective thickness.

The CID camera is now considered to be suitable for the spectrometer operation. The spectrometers will be incorporated as part of existing diagnostics at several locations on the NIF target chamber. All cameras will be calibrated using an extension of the procedure. It will extend to lower X-ray energies using the Manson source and measure the sensitivity variation of the CID over the full pixel array.

\section{CONCLUSION AND FUTURE WORK}

This paper describes the procedures developed to calibrate quantitatively various types of X-ray imaging devices. The procedures were developed using two stable X-ray sources, HEX and MRXS, described in this paper and cover the energy range from $500 \mathrm{eV}$ to $115 \mathrm{keV}$. The heart of the method calibrates the detectors used to measure the source intensity to NIST traceable standards. The method uses radioactive sources and a synchrotron to calibrate photodiodes and energy dispersive detectors. The calibrated detectors allow us to measure the source intensities to an accuracy of several percent.

The sources can then be used to calibrate X-ray imaging devices for both average quantum efficiency and the variation in the efficiency over the detector area. The MRXS has been used to measure the quantum efficiency and its variation for CCD cameras, both front illuminated and back thinned and back illuminated cameras, for the energy range from $710 \mathrm{eV}$ to $8 \mathrm{keV}$. The quantum efficiency measurements have been extended to $26 \mathrm{keV}$ using the HEX. The quantum efficiency for a CID camera was measured using the HEX in the $10 \mathrm{keV}$ to $15 \mathrm{keV}$ range. The method to extend these measurements down to $1 \mathrm{keV}$ using the MRXS is now being developed. The method to measure quantum efficiency variation using the HEX will be developed in the near future and applied to the various types of X-ray imaging devices at the higher spectral energies.

\section{REFERENCES}

Carbone, J., Zulfiquar, A., Borman, C., Czebiniak, S., \& Ziegler, H. (1998). "Large format CID X-ray image sensors,” Proceedings of SPIE 3301, 90 doi:10.1117/12.304550, Solid State Sensor Arrays: Development and Applications II.

Gottwald, A., Kroth, U., Krumrey, M., Richter, M., Scholze, F., \& Ulm, G. (2006). “The PTB high accuracy spectral responsivity scale in the VUV and x-ray range," Metrologia 43.

Haugh, M.J. \& Schneider, M. (2011). (in process), "Quantitative Measurements of X-ray Energy," in Photodiodes-Communications, Bio-Sensing, Measurements and High Energy Physics, InTech Publisher, www.intechweb.org.

International Radiation Detectors (IRD) (n.d.), available from http://www.irdinc.com/axuvhighnrg.html

Janesick, J. (2000). Scientific Charge-Coupled Devices, SPIE Press, Bellingham, WA.

Knoll, G. F. (2001). Radiation Detection and Measurement, $3^{\text {rd }}$ edition (John Wiley \& Sons). 
Maddox, B. et al (2011). "High-energy backlighter spectrum measurements using calibrated image plates," Review of Scientific Instruments 82, 023111.

Marshall, F. J., Ohki, T., McInnis, D., Ninkov, Z., Carbone, J. (2001). "Imaging of laser-plasma x-ray emission with charge-injection devices," Review of Scientific Instruments 72, 713.

Physikalisch-Technische Bundensanstalt (PTB) (n.d.), available at http://www.ptb.de/index_en.html

Poletto, L., Boscolo, A., \& Tondello, G. (1999), "Characterization of a Charge-coupled Detector in the 1100-0.14 nm (1 eV to $9 \mathrm{keV})$ Spectral Range," Applied Optics, 38.

Quaranta, C., Canali, G., Ottavani, G., \& Zanio, K. (1969). "Electron-hole Pair Ionization Energy in CdTe between 85K and 350K," Lettere Al Nuovo Dimento, 4, 908-910.

This manuscript has been authored by National Security Technologies, LLC, under Contract No. DE-AC52-06NA25946 with the U.S. Department of Energy. The United States Government retains and the publisher, by accepting the article for publication, acknowledges that the United States Government retains a non-exclusive, paid-up, irrevocable, world-wide license to publish or reproduce the published form of this manuscript, or allow others to do so, for United States Government purposes.

This work performed under the auspices of the U.S. Department of Energy by Lawrence Livermore National Laboratory under Contract DE-AC52-07NA27344. 